Palavras chave: madeiras tropicais painel colado lateralmente acetato de polivinila emulsão polimérica de isocianato

Histórico:

Recebido 07/04/2014 Aceito 10/08/2015

Keyworks: tropical wood edge glued panel polyvinyl acetate polymeric emulsion of isocyanate

Correspondence: setsuo@ufpr.br
Setsuo Iwakiri', Rosilani Trianoski', Claudete Catanhede do Nascimento², Claudio Gumane', Elaine Cristina Lengowski', Felipe Zatt Schardosin', Rafael Azambuja'

\section{RESISTÊNCIA DAS JUNTAS COLADAS DE MADEIRAS DE Inga alba (SW) Willd E Swartzia recurva Poepp}

RESUMO: O objetivo deste trabalho foi avaliar a qualidade das juntas coladas de madeira de Inga alba e Swartzia recurva utilizando adesivos a base de acetato de polivinila (PVAc) e emulsão polimérica de isocianato (EPI) em gramaturas de 150 e $200 \mathrm{~g} \cdot \mathrm{m}^{-2}$. A resistência das juntas coladas foi avaliada por meio de ensaios de cisalhamento, com base nos procedimentos descritos na norma EN 13354 (2008). O aumento na gramatura não influenciou nos resultados de cisalhamento, indicando os benefícios em termos econômicos com a redução no consumo do adesivo. As juntas coladas com madeira de Swartzia recurva com o adesivo EPI, nas duas gramaturas avaliadas, atingiram o valor mínimo de 2,5 MPa referente ao $5^{\circ}$ percentil inferior estabelecido pela norma EN 13353 (2008). Os resultados demonstraram a viabilidade de uso da madeira de Inga alba e Swartzia recurva para produção de painéis colados lateralmente - EGP, com adesivo EPI e gramatura de $150 \mathrm{~g} \cdot \mathrm{m}^{-2}$.

\section{STRENGTH OF GLUED JOINTS OF Inga alba (SW) Willd AND Swartzia recurva Poepp WOOD}

ABSTRACT: The objective of this study was to evaluate the quality of wood bonded joints of Inga alba and Swartzia recurva using polyvinyl acetate (PVAc) and polymeric emulsion of isocyanate (EPI) in weights of 150 and $200 \mathrm{~g} \cdot \mathrm{m}^{-2}$. The strength of the bonded joints was evaluated through shear tests, using the procedures described in EN I 3354 (2008). The increase in weight did not influence the results of shear strength, indicating the benefits in economic terms with the reduction in the consumption of adhesive. Joints glued with the wood species of Swartzia recurve with EPI adhesive, at the two weights have reached the minimum value of $2.5 \mathrm{MPa}$ concerning the lower 5th percentile established by EN 13353 (2008). The results demonstrated the feasibility of using wood of Swartzia recurva to produce edge glued panels - EGP, with EPI adhesive in weight of $150 \mathrm{~g} \cdot \mathrm{m}^{-2}$. 


\section{INTRODUÇÃO}

A Amazônia brasileira apresenta grande diversidade de espécies florestais com potencial madeireiro, entretanto, poucas espécies são utilizadas comercialmente, conduzindo à exploração bastante seletiva da floresta com impactos negativos ao eco-sistema florestal. Pesquisas visando ampliar a gama de espécies para uso industrial, de forma a otimizar a exploração florestal em regime de manejo sustentado, tornam-se necessárias e essenciais para o desenvolvimento regional na Amazônia.

Dentre as inúmeras espécies com potencial madeireiro, ainda desconhecidas comercialmente, estão as espécies Swartzia recurva Poepp e Inga Alba (Sw) Wild.

A Swartzia recurva é uma das espécies pouco pesquisadas, conhecida vulgarmente como muiragiboia, muiragiboia amarela, pirauichie e gumbeira. São árvores com grandes dimensões e podem ser encontradas em quase toda a Amazônia brasileira, principalmente nos estados do Amazonas, Pará e Rondônia. A sua madeira é dura e pesada, com densidade em torno de $\mathrm{I}, 0 \mathrm{~g} \cdot \mathrm{m}^{-3}$, com cerne de castanho escuro a castanho arroxeado, algumas vezes o alburno amarelo claro se entrelaça com o cerne formando desenhos atrativos; grã regular, textura média, madeira de fácil trabalhabilidade e bom acabamento superficial (LOUREIRO; RODRIGUES, 1979).

A espécie Inga alba é encontrada nas regiões norte, nordeste e centro oeste do Brasil, e é conhecida com nomes vulgares de: ingá, inga-chichí, ingaxixi, inga-xixica, inga-turi, ingá-vermelha e ingá-titica. A sua madeira tem cor róseo-esbranquiçada, textura grossa e é relativamente leve. É destinada para caixotaria, andaimes, lenha, carvão e carpintarias em geral (ALMEIDA et al., 1998).

A utilização da madeira na forma sólida apresenta algumas limitações como dimensões, anisotropia, defeitos naturais que afetam as propriedades físicas e mecânicas das peças. Por meio da tecnologia de colagem de madeiras com emprego de adesivos é possível fabricar produtos reconstituídos de madeira, com maiores dimensões, maior estabilidade dimensional e melhor distribuição da resistência mecânica, com ganhos em qualidade e melhor relação custo-benefício (IWAKIRI, 2005).

Dentre os produtos reconstituídos encontramse os painéis de madeira colados lateralmente ou Edge glued panel - EGP. O "EGP" é um painel composto por sarrafos obtidos a partir da madeira serrada e unido por meio de colagem lateral, podendo ou não ser unidos no topo por emendas do tipo "finger joint" (TIENNE et al., $20 \mathrm{II}$ ). Dois tipos de adesivos são utilizados na produção de painéis EGP, o poliacetato de vinila (PVAc) e emulsão polimérica de isocianato (EPI).

O PVAc é resultante da polimerização (em dispersão aquosa) do acetato de vinila. De acordo com Lopes (2008) este adesivo é popularmente conhecido como "cola branca", sendo muito utilizado pela indústria da madeira e móveis para aplicações de uso interior. $\mathrm{O}$ produto colado com PVAc apresenta alta resistência mecânica em ambiente seco, porém limitações de uso em ambientes com altas temperaturas e umidade relativa (PIZZI, 1983).

A emulsão polimérica de isocianato (EPI) é um adesivo bicomponente, formado por uma base de poliacetato de vinila e um isocianato polimérico (difenilmetano-diisocianato - MDI). Este último tem a função de catalisar a reação de cura do adesivo que ocorre através da reação dos grupos isocianatos com as hidroxilas da madeira. Devido à elevada reatividade, o tempo de aplicação deve ser ajustado de acordo com a recomendação do fabricante (LOPES, 2008).

A colagem de duas peças de madeira depende de vários fatores como estrutura anatômica, massa específica e porosidade. Iwakiri (2005) relata que as propriedades anatômicas da madeira estão relacionadas diretamente com as ações de mobilidade do adesivo para $\circ$ interior da estrutura da madeira, e formação de "ganchos" de ligação entre as faces de colagem da madeira. A porosidade tem uma relação inversa com a massa específica, ou seja, madeira com alta massa específica possui menos espaços vazios e, consequentemente, dificulta a penetração do adesivo no interior da madeira, diminuindo o ancoramento e resultando na baixa adesão mecânica.

Outros fatores como $\mathrm{pH}$ e extrativos presentes na madeira são também importantes, podendo interferir na cura do adesivo durante o processo de prensagem do painel. $\mathrm{O} \mathrm{pH}$ da madeira está situado entre 3,0 e 5,5 e é importante propriedade para todos os tipos de adesivos, pois a madeira tem uma capacidade tampão muito grande que pode interferir com um ácido ou base do sistema catalisado. Entretanto a sua influência é mais significativa nas prensagens a quente. Já com relação aos 
extrativos, dependendo da espécie e da condição de secagem da madeira, pode ocorrer a migração e aumento na concentração dos extrativos na superfície da madeira, prejudicando a formação da ligação adesiva (PIZZI, 1994).

Visando avaliar o potencial de algumas espécies tropicais da Amazônia para produção de painéis colados lateralmente (EGP), este trabalho teve como objetivo analisar a resistência das juntas coladas das madeiras de Inga alba e Swartzia recurva confeccionadas com dois diferentes adesivos e duas gramaturas.

\section{MATERIAL E MÉTODOS}

Foram utilizadas nesta pesquisa madeiras de Inga alba (Sw) Willd e Swartzia recurva Poepp, provenientes da Estação Experimental ZF-2 do Instituto Nacional de Pesquisas da Amazônia, localizada no Município de Manaus, Estado do Amazonas. Para a colagem das peças de madeira foram empregados os adesivos a base de acetato de polivinila (PVAc) e emulsão polimérica de isocianato (EPI).

As madeiras foram obtidas na forma de tábuas e após a secagem ao teor de umidade médio de $12 \%$, foram serradas e aplainadas para obtenção de peças com dimensões finais de 31,0 (comprimento) $x$ 5,0 (largura) x 2,5 cm (espessura), para confecção de juntas coladas.

A massa específica aparente da madeira foi determinada por meio de medições das dimensões e pesos de todas as peças a serem coladas (NBR 7190:1997). As análises químicas foram realizadas a partir de cavacos obtidos das tábuas, com base nos procedimentos descritos na norma TAPPI 204 om-88 para teor de extrativos totais, TAPPI 211 om-93 para teor de cinzas e, TAPPI 252 om-02 para pH.

$O$ delineamento experimental consistiu em duas espécies, dois adesivos (PVAc e EPI), e duas gramaturas ( I 50 e $200 \mathrm{~g} \cdot \mathrm{m}^{-2}$ ), conforme apresentado na Tabela I.

$\mathrm{O}$ adesivo foi aplicado com auxílio de um rolo de espuma sobre uma das faces das peças e a gramatura foi controlada por meio de uma balança de precisão. As duas peças foram prensadas com pressão específica de I $\mathrm{MPa}$ e tempo de prensagem de 3 horas para os dois tipos de adesivos, conforme recomendações dos fabricantes dos adesivos. Foram confeccionadas três juntas coladas por tratamento, totalizando 24 peças.
TABELA 1 Delineamento experimental.

TABLE 1 Experimental design.

\begin{tabular}{lccc}
\hline Espécie & Tratamento & Adesivo & Gramatura $\left(\mathrm{g} \cdot \mathrm{m}^{-2}\right)$ \\
\hline \multirow{4}{*}{ Inga alba } & $\mathrm{I}$ & EPI & 150 \\
& 3 & PVA & 150 \\
& 4 & EPI & 200 \\
& 5 & PVA & 200 \\
\multirow{2}{*}{ Swartzia } & 6 & EPI & 150 \\
recurva & 7 & PVA & 150 \\
& 8 & EPI & 200 \\
& & PVA & 200 \\
\hline
\end{tabular}

Após a colagem e acondicionamento em câmara climática à temperatura de $20 \pm 3^{\circ} \mathrm{C}$ e umidade relativa de $65 \pm 5 \%$, foram confeccionados os corpos-de-prova para ensaios de cisalhamento da linha de cola. Foram testados 20 corpos-de-prova por tratamento, sendo 10 para pré-tratamento a seco e 10 para úmido. No pré-tratamento a úmido, os corpos-de-prova foram submersos em água à temperatura de $20^{\circ} \mathrm{C}$, por um período de 24 horas, antes dos ensaios de cisalhamento. Os ensaios foram conduzidos de acordo com os procedimentos descritos na norma EN I3354/2003 e os resultados foram comparados com os requisitos estabelecidos na norma EN I3353/2003. Após os ensaios de cisalhamento, foram avaliadas as porcentagens de falha na madeira na superfície de ruptura da linha de cola.

Os resultados foram analisados por meio de ANOVA, para identificação de diferença significativa entre os tratamentos propostos e teste de Tukey para comparação de médias. Todos os testes foram efetuados a partir do pacote estatístico Statgraphics VII, a 95\% de probabilidade, e o delineamento estatístico foi o inteiramente casualisado.

\section{RESULTADOS E DISCUSSÃO}

\section{Massa específica aparente e análises químicas}

Os resultados de massa específica aparente e das análises químicas das madeiras de Inga alba e Swartzia recurva estão apresentados na Tabela 2.

O valor médio de massa específica aparente obtido para a madeira de Swartzia recurva foi de 0,983 $\mathrm{g} \cdot \mathrm{cm}^{-3}$, sendo estatisticamente superior em relação à madeira de Inga Alba, cuja média foi de 0,628 g. $\mathrm{cm}^{-3}$. As espécies podem ser consideradas de alta e média massa específica, respectivamente. 
TABELA 2 Resultados de massa específica aparente e análises químicas.

TABLE 2 Results of density and chemical analysis.

\begin{tabular}{lcccc}
\hline Espécie & $\begin{array}{c}\text { Massa } \\
\text { específica } \\
\left(\mathbf{g} \cdot \mathbf{c m}^{-3}\right)\end{array}$ & $\begin{array}{c}\text { Extrativos } \\
\text { totais } \\
\mathbf{( \% )}\end{array}$ & $\mathbf{p H}$ & $\begin{array}{c}\text { Cinzas } \\
\mathbf{( \% )}\end{array}$ \\
\hline Inga alba & $0,628 \mathrm{~b}$ & $4,6 \mathrm{I} \mathrm{b}$ & $5,30 \mathrm{a}$ & $0,35 \mathrm{~b}$ \\
& $(10,16)$ & $(10,7 \mathrm{I})$ & $(\mathrm{I}, \mathrm{II})$ & $(4,32)$ \\
Swartzia & $0,983 \mathrm{a}$ & $6,53 \mathrm{a}$ & $5,24 \mathrm{a}$ & $0,42 \mathrm{a}$ \\
recurva & $(3,15)$ & $(5,27)$ & $(\mathrm{I}, 06)$ & $(5,54)$
\end{tabular}

Médias seguidas de mesma letra na mesma coluna são estatisticamente iguais pelo teste de Tukey a $95 \%$ de probabilidade. Resultados entre parênteses referem-se ao coeficiente de variação.

Cabe ressaltar que, as espécies mais empregadas na produção de painéis colados lateralmente no Brasil são de baixa e média massa específica, como as apresentadas a seguir: Tectona grandis - teca $(0,48$ a 0,64 $\left.\mathrm{g} \cdot \mathrm{cm}^{-3}\right)$, Pinus elliottii e Pinus taeda $\left(0,32\right.$ a $\left.0,34 \mathrm{~g} \cdot \mathrm{cm}^{-3}\right)$, Eucalyptus grandis $\left(0,39 \mathrm{a} 0,5 \mathrm{Ig} \cdot \mathrm{cm}^{-3}\right)$ e Ocotea porosa -

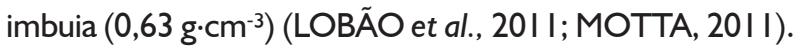

Com relação a análises químicas, a espécie Swartzia recurva apresentou teor de extrativos totais de $6,53 \%$, sendo estatisticamente superior em relação a Inga Alba com valor médio de $4,61 \%$. Não foram constatadas diferenças significativas entre as duas espécies para resultados de $\mathrm{pH}$ e teor de cinzas. Como referência, Bila (2014) obteve para seis espécies de madeiras tropicais da Amazônia, valores de extrativos totais na faixa de $3,19 \%$ a II,67\%.

Os valores médios de $\mathrm{pH}$ de 5,30 e 5,24 obtidos para Swartzia recurva e Inga Alba, respectivamente, estão dentro da faixa de 3,0 e 5,5 mencionado por Iwakiri (2005). Com relação ao teor de cinzas, os valores obtidos para Swartzia recurva e Inga Alba, foram respectivamente de $0,35 \%$ e $0,42 \%$. Bila (2014) obteve para seis espécies de madeiras tropicais da Amazônia, valores de $\mathrm{pH}$ na faixa de 3,99 a 5,40 e teor de cinzas de $0,19 \%$ a $0,70 \%$.

\section{Resultados de cisalhamento das juntas coladas - teste seco}

Os resultados dos ensaios de cisalhamento e percentagens de falhas na madeira das juntas coladas no teste seco estão apresentados na Tabela 3.
TABELA 3 Resultados de cisalhamento das juntas coladas teste seco.

TABLE 3 Results of shear strength of glued joints - dry test.

\begin{tabular}{|c|c|c|c|}
\hline Tratamento & $\begin{array}{c}\text { Cisalhamento } \\
\text { (MPa) }\end{array}$ & $\begin{array}{c}\text { Falhas } \\
(\%)\end{array}$ & $\begin{array}{l}5^{\circ} \text { percentile } \\
\text { inferior }(\mathrm{MPa})\end{array}$ \\
\hline $\begin{array}{l}\text { TI - Inga alba } \\
(\mathrm{EPI} / \mathrm{I} 50)\end{array}$ & $\begin{array}{l}12,18 \text { bc } \\
(13,19)\end{array}$ & 87,00 & 9,11 \\
\hline $\begin{array}{l}\text { T2 - Inga alba } \\
\text { (PVA / I50) }\end{array}$ & $\begin{array}{l}10,99 c \\
(11,35)\end{array}$ & 24,33 & 8,97 \\
\hline $\begin{array}{l}\text { T3 - Inga alba } \\
(\mathrm{EPI} / 200)\end{array}$ & $\begin{array}{l}12,9 \mid \text { bc } \\
(11,31)\end{array}$ & $4 I, 00$ & $|0,5|$ \\
\hline $\begin{array}{l}\text { T4 - Inga alba } \\
\text { (PVA / 200) }\end{array}$ & $\begin{array}{l}10,13 c \\
(16,50)\end{array}$ & 43,33 & 8,06 \\
\hline $\begin{array}{l}\text { T5 - Swartzia } \\
\text { recurva } \\
(\mathrm{EPI} / \mathrm{I} 50)\end{array}$ & $\begin{array}{l}15,08 \mathrm{a} \\
(15,97)\end{array}$ & 72,33 & 11,13 \\
\hline $\begin{array}{l}\text { T6 - Swartzia } \\
\text { recurva } \\
\text { (PVA / I50) }\end{array}$ & $\begin{array}{c}13,90 \mathrm{ab} \\
(13,24)\end{array}$ & 60,33 & 10,90 \\
\hline $\begin{array}{l}\text { T7 - Swartzia } \\
\text { recurva } \\
(\text { EPI / 200) }\end{array}$ & $\begin{array}{l}|5,3| \mathrm{a} \\
(10,60)\end{array}$ & 88,67 & 13,20 \\
\hline $\begin{array}{l}\text { T8 - Swartzia } \\
\text { recurva } \\
\text { (PVA / 200) }\end{array}$ & $\begin{array}{l}|4,0| \mathrm{ab} \\
(\mid 4,87)\end{array}$ & 83,33 & 10,77 \\
\hline
\end{tabular}

Médias seguidas de mesma letra na mesma coluna são estatisticamente iguais pelo teste de Tukey a 95\% de probabilidade. Resultados entre parênteses referem-se ao coeficiente de variação.

O maior valor médio de cisalhamento (15,31 $\mathrm{MPa}$ ) foi obtido para juntas coladas de Swartzia recurva com adesivo EPI e gramatura de $200 \mathrm{~g} \cdot \mathrm{m}^{-2}$, e o menor valor médio (10,13 MPa) obtido para juntas coladas de Inga Alba com adesivo PVA e gramatura de $200 \mathrm{~g} \cdot \mathrm{m}^{-2}$.

Os valores obtidos nesta pesquisa foram superiores aos apresentados na literatura para madeiras de eucalipto, pinus e algumas espécies de madeiras tropicais. Vital et al. (2006) encontraram para madeiras de Eucalyptus saligna, Eucalyptus grandis e Pinus elliottii, coladas com PVAc, valores de resistência ao cisalhamento de 10,24, 9, 12 e 5,40 MPa, respectivamente. Iwakiri et al. (20I3) encontraram para madeiras de Eucalyptus benthamii coladas com PVAc, valor médio de cisalhamento de 9,04 $\mathrm{MPa}$. Com relação às juntas coladas com adesivo EPI, Almeida (2013) obteve valores de 8,42 MPa para Pterogyne nitens (Amendoim), 0,37 MPa para Tabebuia spp. (Ipê), e 6,42 MPa para Hymenaea spp. (Jatobá). 
A madeira de Swartzia recurva apresentou maiores valores médios de resistência das juntas coladas em comparação a madeira de Inga Alba. Para todos os tratamentos com diferentes adesivos e gramaturas, os resultados foram estatisticamente significativos. Este resultado demonstra que a maior massa específica da madeira Swartzia recurva contribuiu para o aumento na resistência das juntas coladas. Por outro lado, o maior teor de extrativos totais presentes na madeira de Swartzia recurva não afetou negativamente a resistência das juntas coladas.

Com relação ao tipo de adesivo, para ambas as espécies não foram constatadas diferenças estatisticamente significativas entre as juntas coladas com EPI e PVA. Entretanto, em termos de médias absolutas as juntas coladas com adesivo EPI apresentaram valores ligeiramente superiores em relação às juntas coladas com adesivo PVA.

Não foram constatadas também diferenças significativas entre as gramaturas de $150 \mathrm{~g} \cdot \mathrm{m}^{-2}$ e $200 \mathrm{~g} \cdot \mathrm{m}^{-2}$ para as duas espécies estudadas. Este resultado é importante em termos de relação custo benefício, tendo em vista a possibilidade de colagem com menor consumo de adesivo.

Quanto à percentagem de falhas na madeira, os valores variaram entre $24,33 \%$ a $88,67 \%$. Plaster et al. (2008) encontraram para juntas coladas de madeiras de Eucalyptus spp. com adesivo PVAc, percentagem entre $26 \%$ a $91 \%$. Iwakiri et al. (2013) encontraram para juntas coladas de Eucalyptus benthamii falhas na madeira de $35,97 \%$. Bila (2014) obteve para juntas coladas de seis espécies de madeiras tropicais, com adesivo EPI, percentagem de falhas na madeira na faixa de $18,75 \%$ a $75 \%$.

Os valores de $5^{\circ}$ percentil inferior variaram de 8,06 MPa a I3,20 MPa. Os valores são considerados altos, entretanto a norma EN considera como requisito para avaliação qualitativa das juntas coladas os ensaios submetidos ao pré-tratamento úmido.

\section{Resultados de cisalhamento das juntas coladas - teste úmido}

Os resultados dos ensaios de cisalhamento e percentagens de falhas na madeira das juntas coladas no teste úmido, após imersão em água à temperatura de $20^{\circ} \mathrm{C}$ por 24 horas, estão apresentados na Tabela 4.

O maior valor médio no teste úmido $(8,4 \mathrm{I} \mathrm{MPa})$ foi obtido para juntas coladas de Swartzia recurva com adesivo EPI e gramatura de $200 \mathrm{~g} \cdot \mathrm{m}^{-2}$, e o menor valor médio (2,52 $\mathrm{MPa}$ ) obtido para Inga Alba com adesivo PVA e gramatura de $200 \mathrm{~g} \cdot \mathrm{m}^{-2}$.

Existem poucas informações na literatura sobre resultados de ensaios de cisalhamento das juntas coladas após pré-tratamento a úmido. Almeida (2013) obteve para algumas espécies de madeiras tropicais coladas com adesivo PVAc e EPI, os seguintes resultados de cisalhamento: Pterogyne nitens (Amendoim) - 0,82 MPa (PVAc) e 5,23 MPa (EPI), tabebuia spp. (Ipê) - 0,35 MPa (PVAc) e I,30 MPa (EPI), e Hymenaea spp. (Jatobá) - 0,76 MPa (PVAc) e 6,42 MPa (EPI). Os valores obtidos nesta pesquisa foram satisfatórios em comparação aos valores apresentados por Almeida (2013), principalmente para as juntas coladas com o adesivo PVAc.

TABELA 4 Resultados de cisalhamento das juntas coladas teste úmido.

TABLE 4 Results of shear strength of glued joints - wet test

\begin{tabular}{|c|c|c|c|}
\hline Tratamento & $\begin{array}{l}\text { Cisalhamento } \\
\text { (MPa) }\end{array}$ & $\begin{array}{c}\text { Falhas } \\
(\%)\end{array}$ & $\begin{array}{l}5^{\circ} \text { percentile } \\
\text { inferior }(\mathrm{MPa})\end{array}$ \\
\hline $\begin{array}{l}\text { TI - Inga alba } \\
(\mathrm{EPI} / \mathrm{I} 50)\end{array}$ & $\begin{array}{c}7,06 \mathrm{a} \\
(29,27)\end{array}$ & 9,67 & 4,02 \\
\hline $\begin{array}{l}\text { T2 - Inga alba } \\
\text { (PVA / I50) }\end{array}$ & $\begin{array}{l}3,09 \text { b } \\
(44,06)\end{array}$ & 0,67 & 1,97 \\
\hline $\begin{array}{l}\text { T3 - Inga alba } \\
(\mathrm{EPI} / 200)\end{array}$ & $\begin{array}{r}7,58 \mathrm{a} \\
(25,28) \\
\end{array}$ & 3,00 & 4,17 \\
\hline $\begin{array}{l}\text { T4 - Inga alba } \\
\text { (PVA / 200) }\end{array}$ & $\begin{array}{c}2,91 \mathrm{~b} \\
(20,03)\end{array}$ & 2,00 & 2,04 \\
\hline $\begin{array}{l}\text { T5 - Swartzia } \\
\text { recurva } \\
(\text { EPI / I 50) }\end{array}$ & $\begin{array}{r}7,58 \mathrm{a} \\
(34,10)\end{array}$ & 38,67 & 3,38 \\
\hline $\begin{array}{l}\text { T6 - Swartzia } \\
\text { recurva } \\
(\mathrm{PVA} / \mathrm{I50})\end{array}$ & $\begin{array}{c}2,70 \text { b } \\
(29,57)\end{array}$ & 0,00 & 1,76 \\
\hline $\begin{array}{l}\text { T7 - Swartzia } \\
\text { recurva } \\
(E P I / 200)\end{array}$ & $\begin{array}{c}8,41 \text { a } \\
(32,81)\end{array}$ & 32,33 & 3,48 \\
\hline $\begin{array}{l}\text { T8 - Swartzia } \\
\text { recurva } \\
\text { (PVA / 200) }\end{array}$ & $\begin{array}{c}2,52 b \\
(40,65)\end{array}$ & 0,00 & $\mathrm{I}, 48$ \\
\hline
\end{tabular}

Médias seguidas de mesma letra na mesma coluna são estatisticamente iguais pelo teste de Tukey a 95\% de probabilidade. Resultados entre parênteses referem-se ao coeficiente de variação.

Não foram constatadas diferenças significativas para os resultados de cisalhamento entre as espécies 
Swartzia recurva e Inga Alba, para todos os tratamentos analisados. Portanto, pode-se afirmar que, para os ensaios após pré-tratamento a úmido, a maior massa específica e teor de extrativos totais da espécie Swartzia recurva não contribuiu para o aumento nos resultados de cisalhamento das juntas coladas.

Com relação ao tipo de adesivo, as juntas coladas com adesivo EPI apresentaram médias de cisalhamento estatisticamente superiores em relação às juntas coladas com PVAc. Este resultado demonstra que o adesivo EPI é mais resistente a umidade, sendo indicado para uso em ambientes com alta umidade relativa. Pizzi (1983) afirma que os produtos colados com adesivo PVAc apresentam baixa resistência da ligação adesiva quando expostos à alta umidade por um período prolongado de tempo. Ainda segundo o mesmo autor, a cura do adesivo PVAc ocorre por meio da evaporação do solventes (água), resultando numa colagem de baixa resistência à temperatura e umidade. Já a cura do adesivo EPI ocorre pela reação de seus grupos de isocianato com os grupos hidroxilas da madeira, promovendo a adesão química mais forte.

Não foram constatadas diferenças significativas entre as gramaturas de $150 \mathrm{~g} \cdot \mathrm{m}^{-2}$ e $200 \mathrm{~g} \cdot \mathrm{m}^{-2}$, sendo este resultado importante sobre ponto de vista econômico, pela possibilidade de colagem com menor consumo de adesivo. Entretanto, esta afirmativa deve ser confirmada por meio de colagem industrial.

Quanto à percentagem de falhas na madeira, os valores obtidos no teste úmido foram muito baixos, com exceção para juntas coladas de Swartzia recurva com adesivo EPI, com percentagem de $38,67 \%$ e $32,33 \%$. Bila (2014) obteve também para juntas coladas de seis espécies de madeiras tropicais com adesivo EPI, baixa percentagem de falhas na madeira, com valores variando na faixa de $3,3 \%$ a $33,3 \%$.

O $5^{\circ}$ percentil inferior é um parâmetro importante de avaliação da qualidade das juntas coladas após pré-tratamento a úmido. Os valores obtidos neste estudo variaram de I,48 $\mathrm{MPa}$ a 4, I7 MPa. Todos os resultados obtidos para as juntas coladas com adesivo EPI, para as duas espécies estudadas, atingiram o requisito mínimo de 2,5 MPa estabelecido pela norma EN I 3353 (2008). Por outro lado, nenhum resultado obtido para as juntas coladas com adesivo PVA atingiram este requisito. Como referência, Almeida (20I3) encontrou para juntas coladas de Pterogyne nitens (Amendoim), Tabebuia spp. (Ipê) e Hymenaea spp. (Jatobá), com adesivo EPI, valores de $5^{\circ}$ percentil de 3,42, 0,09 e 0,28, respectivamente.

\section{CONCLUSÕES}

As juntas coladas com a madeira de Swartzia recurva apresentaram maior resistência ao cisalhamento em comparação à madeira de Inga Alba para o teste seco. Entretanto, para o pré-tratamento a úmido, não foram constatadas diferenças significativas entre as duas espécies.

Tanto para o teste seco, quanto para o úmido, o adesivo EPI apresentou melhor desempenho em relação ao PVA; nos ensaios após pré-tratamento a úmido apenas o adesivo EPI atingiu os valores mínimos de $5^{\circ}$ percentil inferior estabelecido pela norma EN I 3353 (2008).

Não houve incremento nos resultados de cisalhamento das juntas coladas com o aumento na gramatura de $150 \mathrm{~g} \cdot \mathrm{m}^{-2}$ para $200 \mathrm{~g} \cdot \mathrm{m}^{-2}$.

Os resultados demonstraram a viabilidade de uso da madeira de Inga Alba e Swartzia recurva para produção de painéis colados lateralmente (EGP), com adesivo EPI e gramatura de $150 \mathrm{~g} \cdot \mathrm{m}^{-2}$.

\section{AGRADECIMENTOS}

Os autores expressam seus agradecimentos ao INCT - Madeiras da Amazônia, às empresas Momentive S.A. e Henkel Ltda., pelas doações de madeiras e adesivos utilizados nesta pesquisa.

\section{REFERÊNCIAS}

ALMEIDA, S. P.; PROENÇA, C. E. B.; SANO, S. M.; RIBEIRO, J. F. Cerrado: espécies vegetais úteis. Planaltina: EMBRAPA-CPAC, 1998. 464p.

ALMEIDA, V. C. Avaliação do potencial de uso de resíduos de madeira tropical para produção de painéis colados lateralmente - EGP. 20I3. I23 p. Tese (Doutorado em Ciências Florestais) - Universidade Federal do Paraná, Curitiba.

ASSOCIAÇÃO BRASILEIRA DE NORMAS TÉCNICAS. NBR 71 90: Projeto de estruturas de madeira. São Paulo, 1997. 107p.

BILA, N. F. Avaliação da qualidade de juntas coladas de seis espécies de madeiras tropicais da Amazônia. 20I4. 74 p. Dissertação (Mestrado em Ciências Florestais) - Universidade Federal do Paraná, Curitiba. 
EUROPEAN COMMITTEE FOR STANDARDIZATION. EN 13353: Solid Wood panels (SWP) - Requirements. Bruxelas, 2008. I3p.

\section{EUROPEAN COMMITTEE FOR STANDARDIZATION. EN} I3354: Solid Wood panels (SWP) - bonding quality. Bruxelas, 2008. 10p.

IWAKIRI, S. Painéis de Madeira reconstituída. Curitiba: FUPEF, 2005. 247 p.

IWAKIRI S.; TRIANOSKI, R.; CUNHA, A. B.; PRATA, J. G.; HARA, M.; BILA, N. F.; LUIS, R. C. G.; ARAÚJO, R. D.; VILLAS BÔAS, B. T. Avaliação da resistência de juntas coladas da madeira de Eucalyptus benthamii com diferentes adesivos e faces de colagem. Scientia Forestalis, Piracicaba, v. 41 , n. 99, p. $411-416,2013$.

LOBÃO, M. S.; CASTRO, V. R. de; RANGEL, A.; SARTO, C.; TOMAZELLO FILHO, M.; SILVA JÚNIOR, F. G. da; CAMARGO NETO, L. de; BERMUDEZ, M. A. R. C. Agrupamento de espécies florestais por análises univariadas e multivariadas das características anatômica, física e química das suas madeiras. Scientia Forestalis, Piracicaba, v. 39, n. 92 , p. $469-477,2011$.

LOPES, M. C. Espectroscopia no infravermelho próximo aplicada na avaliação de painéis de madeira colados lateralmente. 130 p. Tese (Doutorado em Ciências Florestais) - Universidade Federal do Paraná, Curitiba, 2008.
LOUREIRO, A. A.; RODRIGUES, W. A. Estudo anatômico da madeira do gênero Swartzia (Leguminosae) da Amazônia I. Acta Amazonica, v. 5, n. I, p. 79-86, 1975.

MOTTA, J. P. Propriedades tecnológicas da Madeira de Tectona grandis L.f. proveniente do Vale do Rio Doce, Minas Gerais. 90 p. Dissertação (Mestrado em Ciências Florestais) - Universidade Fedeal de Espírito Santo. Jerônimo Monteiro, 201 I.

PIZZI, A. Wood adhesives: Chemistry and Technology. New York: Marcel Dekker, 1983. 364 p.

PLASTER, O. B.; OLIVEIRA, J. T. S.; ABRAHÃO, C. P.; BRAS, R. L. Comportamento de juntas coladas da madeira serrada de Eucalyptus sp. Cerne, Lavras, v. I4, n. 3, p. 25I-258, 2008.

TECHNICAL ASSOCIATION OF THE PULP AND PAPER INDUSTRY. TAPPI: Tappi Standard Method. Atlanta, USA. Cd-Rom, 2002 (coletânea de normas).

TIENNE, D. L. C.; NASCIMENTO, A. M.; GARCIA, R. A.; SILVA, D. B. Qualidade de adesão de juntas de madeira de Pinus coladas em condições simuladas de serviço interna e externa. Floresta e Ambiente, Seropédica, v. I8, n. I, p. 16-29, 2011.

VITAL, B. R.; MACIEL, A. S.; DELLA LUCIA, R. M. Qualidade de juntas coladas com lâmnas de madeiras de três regiões do tronco de Eucalyptus grandis, Eucalyptus saligna e Pinus elliottii. Árvore, Viçosa, v. 30, n. 4, p. 637-644, 2006. 